\title{
Essais
}

Revue interdisciplinaire d'Humanités

$8 \mid 2016$

Erreur et création

\section{Du potentiel créateur de l'erreur dans les arts et les humanités}

Avant-propos

Myriam Metayer et François Trahais

\section{OpenEdition}

\section{Journals}

Édition électronique

URL : http://journals.openedition.org/essais/4882

DOI : 10.4000 /essais.4882

ISSN : 2276-0970

Éditeur

École doctorale Montaigne Humanités

Édition imprimée

Date de publication : 15 mars 2016

Pagination : 8-17

ISBN : 978-2-9544269-7-6

ISSN : $2417-4211$

\section{Référence électronique}

Myriam Metayer et François Trahais, «Du potentiel créateur de l'erreur dans les arts et les

humanités », Essais [En ligne], 8 | 2016, mis en ligne le 28 octobre 2020, consulté le 31 octobre 2020

URL : http://journals.openedition.org/essais/4882 ; DOI : https://doi.org/10.4000/essais.4882 


\section{Du potentiel créateur de l'erreur dans les arts et les humanités}

\section{Avant-propos}

\section{Myriam Metayer, François Trahais}

En 1962, l'artiste américain Robert Morris réalise Card File. Aujourd'hui conservée au Centre Pompidou à Paris, l'œuvre a de quoi déconcerter le spectateur non averti. Elle se compose de tiroirs métalliques montés sur une planche de bois. Disposé verticalement, l'ensemble contient quarante-huit fiches cartonnées, indexées et rangées par ordre alphabétique ${ }^{1}$. L'artiste y dresse l'inventaire de la totalité des étapes de l'élaboration de l'œuvre en question, y compris des erreurs qu'il a commises. Ainsi observe-t-on dès la première fiche intitulée Accidents (Accidents) la reconnaissance des faux-pas qui ont ponctué l'activité créatrice. La fiche Losses (Pertes) recense les éléments égarés. Enfin la fiche Mistakes (Erreurs) corrige les fautes d'orthographes, les fautes de frappe, les manques et les absences que l'on peut remarquer dans l'ensemble des autres fiches. Robert Morris exploite, met en scène et revendique ses erreurs ${ }^{2}$. Loin le temps où l'académie enseignait de ne laisser aucune trace de pinceau sur la toile ; où, lisse, la surface ne devait rien laisser paraître des hésitations, des maladresses ou des repentis de l'artiste. Card File non seulement refoule l'esthétique classique mais aussi se détourne de la conception traditionnelle de l'acte de création. Autoréférentielle, l'œuvre échappe à toute appréciation stylistique et se ferme à toute contemplation. Dans le sillage des avant-gardes historiques, Robert Morris met à mal les critères et les valeurs de l'art qui dominaient depuis la Renaissance : beauté, respect des rudiments du métier, qualité plastique. L'œuvre est désormais envisagée comme le produit d'un enchaînement d'idées et de décisions au cour desquelles l'erreur devient un élément dynamique du processus de création.

1 Robert Morris, Card File (Fichier), 11 juillet 1962 - 31 décembre 1962. Matériaux divers (métal, bois, papier), 68,5 × 27 × $4 \mathrm{~cm}$. Collection Centre Pompidou.

2 Ajoutons que Card File est principalement reconnue pour avoir annoncé certaines logiques spécifiques à l'art conceptuel, à savoir le recours au " langage comme moyen de saisir et de rendre compte de l'action, du faire ", in Michel Bourel, "Robert Morris : commentaire de l'œuvre ", Catalogue Art Conceptuel I, Bordeaux, CAPC éditeur, 1988, p. 25. 
La démarche de Robert Morris participe d'un phénomène plus global qui, à partir des années 1960, touche les arts et les sciences humaines. En termes épistémologiques, ces domaines se sont vus assignés l'élaboration d'une conscience critique et réflexive portant sur leurs propres outils méthodologiques, leurs présupposés idéologiques ainsi que leurs modèles historiographiques ${ }^{3}$. La beauté et la vérité ne sont plus considérées comme des références absolues. Au point qu'en 2004, le magazine d'information de la recherche européenne observe que cette évolution tendrait "à réduire le fossé qui sépare [les artistes et les scientifiques] $»^{4}$. L'erreur n'est pas une fatalité. Beaucoup s'accordent aujourd'hui à le penser ${ }^{5}$. Malgré tout, quel chercheur, au quotidien, trouve l'aisance d'exposer ses propres erreurs ? Comment s'autoriser à se laisser surprendre par l'imprévisible quand celui-ci met à nu la crainte de l'erreur qu'auront croisée, ou que croiseront, peu ou prou nombre d'universitaires au cours de leurs travaux ? Les sciences humaines, au contraire des arts, restent attachées à l'idée que le savoir s'élabore par la mise à jour de résultats de recherche plutôt que par la prise en considération du processus qui mène à ces mêmes résultats. Paru en Italie en 1975, Giochi di pazienza de Carlo Ginzburg et Adriano Prosperi demeure un exemple de publication notable, mais rare, témoignant des réflexions qu'ont pu développer certains chercheurs au cours de ces quarante dernières années ${ }^{6}$. La démarche des deux historiens n'est pas sans évoquer celle de Robert Morris. Ils présentent leurs résultats tout en suivant le fil chronologique du développement de leurs recherches, non sans problème car étant poussés à devoir décrire leurs erreurs « avec un souci de l'analyse d'habitude réservé à l'exposition de la vérité " ${ }^{7}$. Au début des années 1980, la notion de jeu revient sous la plume d'Edgar Morin dans un article intitulé «Le jeu de la vérité et de l'erreur $»^{8}$ et dans lequel le sociologue et

3 Sur ce point voir notamment : "À quoi servent les sciences humaines ? - Cycle de conférences (janvier 2009-février 2012) ", in Tracés. Revue de Sciences humaines : http://traces.revues. org/543.

4 Commission européenne, "La recherche dans tous ses états ", in Commission européenne, RDT info, Art \& Science, numéro spécial, Bruxelles, mars 2004, p. 19.

5 Depuis quelques années, le rôle positif de l'erreur dans la recherche tend à être réévalué. Parmi les publications les plus notables citons : Jean C. Baudet, Les plus grandes erreurs de la science, Paris, La Boîte à Pandore, 2014 ; Girolamo Ramunni, Les lieux des erreurs scientifiques, Paris, Éditions Le Cavalier Bleu, coll. «Lieux de... », 2012 ; Dominique Rousseau, Michel Morvant (éd.), «L'Erreur », Le Temps des savoirs, Revue interdisciplinaire, $\mathrm{n}^{\circ}$ 2, Paris, Odile Jacob, 2000. Notons que Frédéric Lambert rappelle dans son article certaines de ces références auxquelles il ajoute des ouvrages dans lesquels l'erreur est considérée sous un angle négatif.

6 Carlo Ginzburg, Adriano Prosperi, Giochi di pazienza. Un seminario sul "Beneficio di Cristo", Torino, Einaudi, 1975.

7 Ibid., p. 179 (traduction personnelle) : «Gli stessi nostri errori finivano con l'essere descritti con l'analitica minuzia di solito riservata all'espozione della verità ".

8 Edgar Morin, "Le jeu de la vérité et de l'erreur ", in Université Claude Bernard, L’Erreur, actes du colloque, Lyon, Presses universitaires de Lyon, 1982, p. 113-127. À notre connaissance, 
philosophe envisage la relativité de l'erreur par rapport à la science. Comme dans un jeu, l'erreur révèle la part de hasard qui sillonne le cheminement intellectuel de celui qui la commet. L'erreur serait d'autant plus imprévisible que sa nature est difficilement saisissable. "Faire erreur prend mille formes " nous enseigne Aristote. Ces mille formes, selon le contexte et l'époque, sont celles de la faute, de l'illusion, de l'échec ou bien encore de l'imprécision et de l'égarement - autant de notions auxquelles l'erreur est assimilée. De même que Robert Morris nous invitait à revoir les critères de jugement sur l'art, peut-être devrions-nous repenser la place qu'occupe l'erreur dans toute démarche intellectuelle. En effet, si de nos jours l'erreur est acceptée comme phénomène faisant partie du processus de création, celle-ci peine encore à être reconnue dans l'élaboration des discours sur l'art, et plus largement au sein des sciences humaines.

Les dix articles réunis ici explorent le rôle de l'erreur et son potentiel créateur au sens large du terme, tant du point de vue de la création artistique et littéraire que du point de vue heuristique. Ainsi, le dossier laisse une large place à la réflexion de type herméneutique en interrogeant la portée de l'erreur dans l'élaboration des savoirs et la transmission des connaissances en regard de l'interprétation des arts et de la littérature. Le projet de cette publication fait suite à la rentrée de l'École Doctorale Montaigne Humanités, organisée le 5 novembre 2013, sur le thème "Se tromper, entre errance et liberté ${ }^{10}$. Plusieurs intervenants issus des études littéraires, de la traductologie, de la philologie, de l'histoire et de l'histoire de l'art avaient alors été invités à s'interroger sur le rôle de l'erreur dans leur champ respectif de recherche ainsi qu’à témoigner de sa place dans le quotidien de leurs travaux universitaires. Encouragés par la richesse des discussions - qui portèrent notamment sur la question des bénéfices de l'erreur au sein de l'enseignement des humanités - nous avons commencé, avec les outils conceptuels propres à notre discipline, l'histoire de l'art, à engager une réflexion s'articulant autour de l'erreur et de la création et déclinant les enjeux théoriques de la rencontre entre ces deux notions. L'Université Bordeaux Montaigne a aussi manifesté un intérêt pour l'erreur dans lequel s'inscrit le présent dossier. Rappelons ainsi que le 6 juin 2014, s'est tenue à la Maison des Sciences de l'Homme d'Aquitaine

Edgar Morin est l'un des rares penseurs à avoir souligné, dès le début des années 1980, le rôle fondamental de l'erreur dans l'histoire des productions scientifique et conceptuelle.

9 Aristote, Éthique à Nicomaque, II. 6, 1107 a 14, IVEe siècle av. J.-C. ; traduction de J. Tricot, Paris, Vrin, 1979. Cité par François Rigolot, L’Erreur de la Renaissance. Perspectives littéraires, Paris, Honoré Champion, 2002, p. 8.

10 Se tromper, entre errance et liberté, journée de rentrée de l'École Doctorale Montaigne Humanités, MSHA (Maison des Sciences de l'Homme d'Aquitaine) Université Bordeaux Montaigne, mardi 5 novembre 2013. 
une journée d'étude sur le thème de "L'Erreur " "tandis qu'en décembre de la même année, avait lieu à Bordeaux le colloque international «L'Erreur chez Montaigne » au cours duquel cette notion fut envisagée comme l'un des principaux moteurs des célèbres Essais ${ }^{12}$.

C'est en premier lieu le caractère relatif de la vérité qu'explorent chacun à leur manière les auteurs des articles du dossier : vérité historique, vérité philosophique, vérité fictionnelle constituent autant de variations sur un même thème. Le rationalisme cartésien, qui avec le Discours de la méthode (1637) a fixé pour plusieurs siècles la manière d'envisager la connaissance dans ses termes construits, a associé l'erreur aux deux notions cardinales que sont la vérité et la liberté $^{13}$. Comme le rappelle François Rigolot dans une étude ${ }^{14}$ consacrée à la conception de l'erreur durant la Renaissance, «Descartes reprend la question qu'avait déjà posée Saint Augustin : Si Dieu est la Vérité, qui ne souffre changement ni hésitation, comment a-t-il pu donner aux êtres humains une âme susceptible d'égarements ? [Confessions IV, XV, 26]. Il répond que l'erreur ne vient pas d'un défaut dans le plan divin mais du mauvais usage que nous faisons de notre liberté ${ }^{15}$. Cette affiliation a perduré dans le temps et elle revient communément dans les définitions que nos dictionnaires et encyclopédies actuels proposent de l'erreur, qu'ils concernent les sciences dites dures ou bien celles dites humaines. Pour exemple, on peut lire dans le Dictionnaire d'histoire et philosophie des sciences à l'entrée intitulée "Maîtrise de l'erreur ": "Le plus petit calcul révèle sans fard la place constitutive de l'erreur dans la condition humaine. Condition libre : la liberté de l'homme est engendrée par sa capacité d'erreur, la possibilité de se tromper ouvre des plages de l'auto-

11 L'erreur, journée d'études organisée par Sandy Pecastaing, Isabelle Poulin et Jérôme Roger, MSHA (Maison des Sciences de l'Homme d'Aquitaine), Université Bordeaux Montaigne, vendredi 6 juin 2014. Cette manifestation pluridisciplinaire a accueilli des représentants issus de domaines variés (la littérature, la traduction, l'histoire de l'art, la psychanalyse, les sciences). Nous remercions vivement Sandy Pecastaing de nous avoir invités à participer, en tant que représentants de l'histoire de l'art, à cette journée d'études dont les débats fructueux ont alimenté l'élaboration de ce dossier.

12 L'erreur chez Montaigne, Colloque de la Société Internationale des Amis de Montaigne, Bibliothèque Mériadeck de Bordeaux, 3-5 décembre 2014. Qu’elles soient volontaires ou involontaires, qu'elles relèvent du simulacre ou du mensonge, les erreurs de Montaigne y furent décrites et analysées dans le souci de révéler l'importance de la réparation et de la correction dans la pensée de l'humaniste bordelais.

13 Le rapport de la pensée de Descartes à l'erreur a déjà donné lieu à des publications. Parmi celles-ci nous indiquons : Gaston Mauge, "Sur Descartes et l'erreur ", Revue de l'Enseignement philosophique, 1986, 36, n 3, p. 34-38; Fernand Hallyn, "L'illusion et l'erreur dans le monde de Descartes ", Cuadernos de filologia francesca, n 11, 1999.

14 François Rigolot, L'Erreur de la Renaissance. Perspectives littéraires, op. cit. Nous tenons à signaler l'intérêt de cette étude, l'une des rares, à notre connaissance, à analyser la notion d'erreur dans une double perspective historique et littéraire.

15 Ibid., p. 367-368. 
nomie ${ }^{16}$. Cette définition favorise l'idée que l'être humain et de surcroît le scientifique devraient surmonter l'erreur dans le souci de la recherche de la vérité, invitant à penser que la condition de la liberté résiderait bien dans le droit à l'erreur mais pas, a priori, dans le fait de commettre l'erreur. Ainsi de la possibilité de l'erreur émerge " un nouveau type d'adéquation au "réel" »"

Un retour sur l'analyse linguistique du vocable " erreur " nous incite cependant à porter un regard critique sur l'articulation que la notion qui s'y rattache entretient avec celle de vérité. En comparant les propriétés linguistiques et l'évolution sémantique du nom « erreur » et du verbe " se tromper ", Frédéric Lambert, dont l'article ouvre le dossier, rend compte de l'éventail de leurs significations. Il constate que l'un comme l'autre renvoient particulièrement à l'idée de cheminement. Supposant qu'une recherche est aussi et d'abord un parcours, l'auteur en vient à s'interroger sur la fonction heuristique de l'erreur. "La recherche est la sœur de l'erreur ", conclut-il, dans la mesure où l'une et l'autre relèvent de la métaphore du chemin.

La dimension géographique et intellectuelle de l'errance est justement au cour de l'article d'Hernán González Bordas. Celui-ci retrace l'itinéraire de quatre érudits qui, à partir du XVIII ${ }^{\mathrm{e}}$ siècle, propagèrent la lecture erronée d'une inscription située sur le monument antique des Flauii à Kasserine. Menant son lecteur de l'Espagne à la Tunisie, il montre comment la contingence (la myopie et le bras cassé de Francisco Ximenez, érudit espagnol) a contribué à propager une mauvaise transcription de l'inscription épigraphique. Une fois l'erreur détectée, il revient aux épigraphistes contemporains d'en retracer le parcours afin de retrouver le sens de l'inscription originale. C'est ce à quoi s'attache Hernán González Bordas qui imprime à son texte la forme de l'enquête. Après avoir présenté les hypothèses de la transmission de l'erreur formulées par ses prédécesseurs, il propose sa propre version de la tradition manuscrite de la lecture erronée. Ainsi, en tentant d'objectiver l'erreur et en retraçant l'histoire de cette dernière, l'auteur s'inscrit lui-même dans la filiation des recherches menées par ses prédécesseurs.

Marco Salucci questionne la valeur épistémologique du commentaire philosophique des œuvres d'art. Il revient sur deux épisodes connus des philosophes et des historiens de l'art : l'erreur d'interprétation de Martin Heidegger, dans L'Origine de l'ouvre d'art (1931-1932), à propos de toiles de Vincent Van Gogh représentant des chaussures, et l'erreur commise par Michel Foucault au sujet des Menines de Diego Velazquez dans Les Mots et les choses (1966). Meyer Schapiro et Daniel Arasse, historiens de l'art, ont chacun démontré que les interprétations des deux philosophes sont erronées dans la mesure où elles éloignent les œuvres

16 Stéphane Callens, "Maîtrise de l'erreur ", in Dominique Lecourt (éd.), Dictionnaire d'histoire et philosophie des sciences, Paris, PUF, 2006 (1999), p. 708.

17 Id. 
de la réalité des faits historiques. Mais erreur y a-t-il vraiment ? Certes, sous le regard des philosophes, les tableaux de Velázquez et de Van Gogh se dérobent bel et bien à l'histoire. Mais Marco Salucci en ayant recours aux concepts de différence et de répétition formulés par Gilles Deleuze montre tout l'intérêt de l'interprétation philosophique. Comme il l'explique, en faisant " agir et réagir [les œuvres] de façon différente ", les philosophes élaborent des concepts nouveaux libérant celles-ci « du poids du vrai».

Cassie Bérard, quant à elle, met à l'épreuve l'efficacité de la méthode d'enquête de l'universitaire, écrivain et critique littéraire qu'est Pierre Bayard, selon lequel une vérité empirique existerait dans le monde fictionnel. Sur le mode du tel est pris qui croyait prendre, Cassie Bérard montre les limites de la méthode de Pierre Bayard en l'appliquant à L'emploi du temps, roman de Michel Butor publié en 1956, dont la narration se développe à partir et autour de l'erreur et dont la lecture, comme le précise l'auteure de l'article, repose sur le soupçon et l'incertitude.

Enfin, Nina Mansion, dans une démarche historiographique, examine les enjeux de l'erreur collective et partagée dans le cercle des historiens de l'architecture. Ces derniers, par la particularité de leur objet d'étude, empruntent une méthodologie singulière. L'architecture est le résultat de l'intervention d'acteurs multiples : architectes, bureaux d'études, ingénieurs, entrepreneurs, administratifs, officiels. En tant que processus de création, le projet architectural apparaît comme une pensée collective, loin de ne concerner que le seul " génie » créateur de l'architecte concepteur. Ainsi, tenter de restituer la réalité des faits historiques demande de considérer les sources comme témoignages d'une pensée collective dont il faut explorer les strates.

Dans le sillage de la réflexion déjà portée en 1982 par Edgar Morin, les articles suivants interrogent le rapport à la norme, qu'elle soit discursive ou esthétique :

\begin{abstract}
(...) toute création originale apparaît comme déviance par rapport à une norme et notamment par exemple dans la norme qui était celle du réalisme en peinture. À ce moment-là quelque chose n'obéissant pas à ces normes quasiphotographiques apparaît comme une erreur, une erreur profonde, une erreur de sens. Alors on pose toujours de façon sociologique ou culturelle l'erreur pour qualifier la déviance et l'originalité, pour la condamner. ${ }^{18}$
\end{abstract}

Les enjeux de la norme dans le domaine artistique qu'Edgar Morin tente de saisir ont très largement été explorés par la discipline de l'histoire de l'art. Celle-ci a montré qu'en termes historiques la création artistique a, dans la culture occidentale, toujours été encadrée par des règles et des préceptes, d'ordre formel ou théorique ${ }^{19}$. C'est aussi le cas de la littérature et de la poésie.

18 Edgar Morin, «Le jeu de la vérité et de l'erreur », op. cit., p. 128.

19 La doctrine classique de l'opération esthétique comporte, par exemple, trois éléments permanents d'interprétation de l'œuvre d'art : l'inspiration, le canon, le modèle. 
Raphaelle Longuet, dans un article consacré à la Manière de bien penser dans les ouvrages d'esprit du Père Bouhours (1687), conclut que la vérité est la qualité première que la pensée académique accorde au texte littéraire. De même que dans l'Ars poetica Horace répertoriait " les erreurs à ne pas commettre si l'on veut être bon poète latin ${ }^{20}$, le Père Bouhours compile les défauts de style qui nuisent à l'établissement de la vérité comme base du jugement de goût.

Abordant le problème de la représentation du point de vue esthétique, les articles de Basile Pallas et de Frédéric Le Gouriérec introduisent la question de l'émergence de la modernité en peinture et en littérature. L'étude de Basile Pallas envisage la représentation de la réalité comme un échec, un ratage programmé et une aberration perceptive au sein de quelques romans écrits au XIX ${ }^{\mathrm{e}}$ siècle dont les personnages principaux sont des artistes. La lecture d'Honoré de Balzac, de Guy de Maupassant, d'Émile Zola et des frères Goncourt montre que si la mimesis a pu incarner un modèle et une norme en matière de figuration artistique, celle-ci reste un idéal à atteindre.

Frédéric Le Gouriérec observe l'impact de la modernité artistique occidentale au sein des arts visuels en Chine. Allant du XVIII e siècle à la période actuelle, l'article analyse les influences de l'art européen dans les techniques et les modes de représentation de l'art chinois. Face à l'hégémonie culturelle de l'Europe et des États-Unis, un nouvel académisme s'impose durant l'époque maoïste. La propagande d'État qualifie alors de « contre-révolutionnaire » un art ayant assimilé les principes de la modernité. Il s'agirait d'un " bricolage » selon Frédéric Le Gouriérec, qui aurait fait de l'erreur de style une alternative au réalisme socialiste alors considéré comme la norme. Depuis les années 1990, la persistance d'une culture visuelle paysanne chinoise, dès lors qu'elle est associée au dogmatisme formel de l'art officiel, initie une nouvelle forme de " kitsch », toujours selon l'auteur. Qu'il s'agisse d'un " bricolage » ou d'un objet relevant du " kitsch », l'art contemporain chinois fait se rencontrer l'héritage de la peinture traditionnelle, la modernité occidentale et le dogme de l'art officiel dans une posture plus subversive qu'il n'y paraît, faisant de l'erreur une alternative à toute conception normative de la création artistique. Selon cette idée, les esthétiques divergentes - considérées comme des erreurs par le goût officiel ou dominant, et parfois aussi qualifiées de "caprices ${ }^{21}-$ trouvent dans la notion de bricolage un outil théorique à partir duquel œuvrer. Créer par des moyens détournés c'est bricoler à partir des règles artistiques et, plus large-

20 François Rigolot, L'Erreur de la Renaissance, op. cit., p. 301. François Rigolot aborde en particulier les liens entre erreur et licence poétique.

21 En matière d'histoire de l'art, la question est notamment analysée dans : Francis Haskel, $\mathrm{La}$ norme et le caprice. Redécouvertes en art. Aspect du goût, de la mode et de la collection en France et en Angleterre, 1789-1914, Paris, Flammarion, 1986. Traduit de l'anglais par Robert Fohr (Éd. Originale : Rediscoveries in Art. Some Aspects of Taste, Fashion and collection in England and France, Phaidon Press Limited, 1976). 
ment, des normes sociales et culturelles. Mais la pratique artistique ne saurait se limiter au domaine de l'esthétique. Dans la Pensée Sauvage, Claude LéviStrauss, à qui nous devons la notion de bricolage, explique :

Tout le monde sait que l'artiste tient à la fois du savant et du bricoleur : avec des moyens artisanaux, il confectionne un objet matériel qui est en même temps objet de connaissance. ${ }^{22}$

La comparaison proposée par l'anthropologue est riche de significations. L'artiste serait-il, quelque part, un savant ? Pourrait-on situer sur un même plan théorique la création des œuvres d'art et l'élaboration des objets de connaissance? Se fait jour, ici, l'articulation entre la vérité et la connaissance ; la connaissance autorisant que penser c'est créer tandis que la vérité demande à ce qu’on la dévoile toujours un peu plus. Considérer l'erreur en regard de la création demande de s'interroger sur ce qu'engage cette dernière notion : créer pour conforter la vérité ? Ou bien créer pour produire une connaissance nouvelle ?

Ces questions peuvent s'appliquer autant à l'art qu'aux humanités. Penser ou créer par des moyens détournés c'est bricoler à partir des règles artistiques ou des méthodes scientifiques normées. Mais avec Card File, Robert Morris amène à penser l'erreur comme un nouveau paradigme artistique. Au début des années 1960, il ouvre une voie que d'autres artistes exploreront en traduisant l'erreur en termes plastiques. C'est le sujet que propose d'analyser l'article de François Maheu en démontrant que l'erreur, une fois envisagée comme forme plastique, bénéficie d'une portée théorique inédite. Douglas Huebler et John Baldessari ont marqué l'histoire du conceptualisme en intégrant l'erreur photographique - en tant qu'acte conscient et pleinement assumé - afin de discuter la subjectivité des critères esthétiques de ce médium. Chez Huebler, l'erreur photographique se loge dans le flou alors que chez Baldessari elle perturbe la composition de l'image. Ces erreurs intentionnelles rapprochent les images obtenues des photographies d'amateurs. En cela, elles libèrent le médium des règles du professionnel au profit d'une approche analytique de l'œuvre d'art.

L'article de Claire Labastie aborde les Erratas du plasticien Gilles Barbier, œuvres qui révèlent toute l'absurdité qui réside dans le fait de copier ou plus exactement dans le fait de recopier. Au croisement de l'obstination du scribe Bartleby ${ }^{23}$ et du dessein encyclopédique de Bouvard et Pécuchet ${ }^{24}$, Gilles Barbier recopie les pages du dictionnaire Larousse en commettant, a priori involontairement, assez d'erreurs pour que celles-ci permettent un "point de retour sur l'œuvre ». En effet, les fautes et les oublis enrichissent la démarche de Gilles Barbier d'un supplément de réflexivité et d'humour

22 Claude Levi-Strauss, La pensée sauvage, Paris, Plon, 1962, p. 37.

23 Herman Melville, Bartleby, le scribe. Une histoire de Wall-Street (Bartleby, the Scrivener. A History of Wall-Street), 1853.

24 Gustave Flaubert, Bouvard et Pécuchet, 1881. 
contenu dans une forme plastique, celle de l'Erratum. Si le repeint permet d'effacer l'erreur du peintre, les Erratas de Gilles Barbier révèlent et assument l'aspect forcément erroné de la copie. Là où se situe le " pouvoir générateur " de l'erreur, selon Claire Labastie, la répétition bénéficie d’une dimension créatrice non convenu.

Les dix études réunies ici ramènent l'erreur à une question de point de vue que celui-ci soit de l'ordre du disciplinaire (histoire, philosophie, littérature...), de l'esthétique ou du méthodologique. À vouloir observer l'erreur au prisme de la création, c'est la relativité de l'erreur qui émerge en contrepoint. L'ensemble des études de ce dossier se développe sur les bases d'un raisonnement implicite : l'erreur n'est reconnue qu'en rapport à quelque chose d'autre. Ce quelque chose sera fonction de critères de goût ou de pensée, ou bien encore de normes. L'erreur, dès lors qu'elle est assumée comme partie constituante de la recherche et plus largement de la connaissance, apparente cette dernière à la création littéraire et picturale. Néanmoins, si le processus est comparable, l'objectif et l'intention ne sont certainement pas les mêmes. Cette réflexion invite à nous retourner vers la forme de l'essai - cette double démarche cognitive et narrative se prêtant au jeu de l'incertain et de la subjectivité. Theodor Adorno nous rappelle justement que l'essai, dont l'horizon ne saurait être ni l'art ni la littérature, "s'expose à l'erreur " ${ }^{25}$. En revendiquant le rôle productif de l'erreur à tous les niveaux de la pensée, Edgar Morin s'inscrit dans une perspective similaire :

«L'essai, entre le coup de pinceau et le verbe, n’est pas un chemin improvisé ni arbitraire, il est la stratégie d'une démarche ouverte qui ne dissimule pas sa propre errance, sans pour autant renoncer à saisir la vérité fugace de son expérience $»{ }^{26}$

Au-delà de l'analogie avec l'écriture et la peinture, l'évocation de l'errance fait écho à l'expérience que nous avons menée pendant l'élaboration de ce dossier. Les textes qui suivent émanent de jeunes chercheurs et de chercheurs confirmés issus d'universités européennes et nord-américaines ainsi que de disciplines variées. Mais au-delà de leur diversité, les contributions proposées sont le fruit des errances de leurs auteurs et des conseils de leurs relecteurs.

25 Théodore W. Adorno, «L'Essai comme forme ", in Notes sur la littérature, Paris, Flammarion, coll. Champs, 1999 (1958), p. 13.

26 Edgar Morin, Raùl Motta, Emilio-Roger Cuirana, Eduquer pour l'ère planétaire. La pensée complexe comme méthode d'apprentissage dans l'erreur et l'incertitude humaine, Paris, Balland, 2003, p. 19. 
Dans ce va et vient entre l'art et la norme, entre la science et la méthode, tous ceux qui ont participé au montage de ce dossier ont su jouer leur rôle.

Nous leur en savons gré.

Myriam Metayer

Centre François-Georges Pariset - EA 538

Université Bordeaux Montaigne myriam.metayer@u-bordeaux-montaigne.fr

François Trahais

Centre François-Georges Pariset - EA 538

Université Bordeaux Montaigne

francois.trahais@u-bordeaux-montaigne.fr 